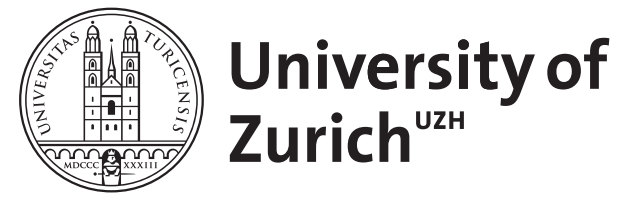

Zurich Open Repository and Archive

University of Zurich

University Library

Strickhofstrasse 39

CH-8057 Zurich

www.zora.uzh.ch

Year: 2014

\title{
Ambulante Antikoagulation - eine Klasse für sich
}

Djalali, S ; Senn, O

Posted at the Zurich Open Repository and Archive, University of Zurich

ZORA URL: https://doi.org/10.5167/uzh-95055

Journal Article

Published Version

Originally published at:

Djalali, S; Senn, O (2014). Ambulante Antikoagulation - eine Klasse für sich. PrimaryCare, 14(2):25. 


\section{Ambulante Antikoagulation - eine Klasse für sich}

\begin{abstract}
Wer hätte das gedacht? Wie guidelinekonform eine Therapie mit oralen Antikoagulanzien gestartet wird, hängt nicht davon ab, ob ein Kardiologe oder Allgemeinmediziner behandelt. Massgeblich ist viel mehr, ob der Arzt im Spital arbeitet, oder in der Praxis tätig ist.
\end{abstract}

Eine Befragungsstudie unter 226 zufällig ausgesuchten Hausärzten, Internisten und Kardiologen im Kanton Zürich brachte erstaunliches zutage: Wie eine Therapie mit oralen Antikoagulanzien eingeleitet wird, variierte beträchtlich wenn man Spitalmediziner mit niedergelassenen Kollegen verglich. Dabei spielte es keine Rolle, welchen Facharzttitel die Mediziner trugen.

\section{Ein Fall für alle Fälle}

Neben der Selbsteinschätzungsfrage, wie häufig sie Guidelines in der täglichen Praxis nutzen, waren die Studienteilnehmer mit einer Fallvignette konfrontiert, in der ein ambulanter Patient mit nichtvalvulärem Vorhofflimmern und hohem Schlaganfallrisiko (CHAD2Score $\geq 2$ ) beschrieben wurde, bei dem gemäss Guidelines eine Langzeitbehandlung mit oralen Antikoagulanzien eingeleitet werden müsste. 93,7\% der Befragten wählten diese Option und verhielten sich damit guidelinekonform, obwohl die Mehrheit der ambulant tätigen Allgemeinmediziner und Internisten Ärzte angab, nur selten Guidelines für sich zu nutzen.

\section{Mehr Guidelines im Spital}

Unter den Spitalärzten war der Anteil von bekenenden Guidelinenutzern dagegen hoch, nur 12,2\% gaben an, kaum Guidelines zu nutzen. Während die Notwendigkeit einer Langzeittherapie von der grossen Mehrheit korrekt erkannt wurde, zeigte sich ein deutlicher Unterschied zwischen Praxis- und Spitalärzten in der Einleitung einer guidelinekonformen oralen Antikoagulanzientherapie. Während 79,7\% der Spitalärzte eine guidelinekonforme Medikation mit entweder niedrigdosierten oralen Vitamin-K Antagonisten (OAK) oder einer überlappenden Kombination aus niedermolekularem Heparin und OAK wählten, war der Anteil bei den ambulant tätigen Kollegen mit korrekter OAK Einleitung (51\%) deutlich geringer. Dieser Unterschied zwischen Spital- und Praxisärzten war unabhängig von der jeweiligen Fachspezialität.

\footnotetext{
Viele Einflüsse im ambulanten Sektor

Allein der Fakt, dass sich ambulant tätige Kollegen weniger streng an die Guidelines zur OAK-Therapie halten, sagt noch nichts über die Qualität ihrer Arbeit aus. Wichtig für die Interpretation der Studienergebnisse sind die unterschiedlichen Charakteristika des ambulanten Sektors gegenüber der Sekundärversorgung im Spital. Man geht davon aus, dass die Guidelineadhärenz von Ärzten mindestens davon abhängt

- wie konsistent die Studiendaten sind, auf denen die Guidelines basieren

- wie vertraut der einzelne Arzt mit den Guidelines ist

- wie sehr die Guidelines mit der eigenen Meinung und Erfahrung des Arztes übereinstimmen
}

- wie gross der erwartete Benefit der guidelinekonformen Behandlung ist

- mit welcher Patientenpopulation der Arzt es zu tun hat

- wie pragmatisch sich die Guidelines umsetzen lassen (Infrastruktur und organisatorisches Umfeld des Arztes)

Es ist zu bedenken, dass Guidelines häufig auf Studiendaten von eher jüngeren Patienten mit einzelnen Erkrankungen (monomorbid) basieren, die Mehrheit der Patienten in der ambulanten Grundversorgung aber älter und multimorbid ist und eine leitliniengerechte Implementierung von therapeutischen Strategien damit nicht immer sinnvoll oder machbar erscheint.

\section{Anpassung an den Alltag}

In dieser Studie spiegelt sich dies in den Befragungsergebnissen der Kardiologen-Subgruppe wider: An sich gaben 90,3\% der niedergelassenen Kardiologen an, generell immer/häufig Guidelines zu nutzen, doch in der Fallvignette wählten nur 58,2\% die guidelinekonforme Initialtherapie. Wer draussen im ambulanten Sektor arbeitet, muss sich bzw. die Auslegung der Guidelines offenbar anpassen.

\section{Literatur}

Gerber B, Stussi G, Rosemann T, Senn O. The health care setting rather than medical speciality impacts on physicians adherence to guideline-conform anticoagulation in outpatients with non-valvular atrial fibrillation: a cross sectional survey. BMC Cardiovasc Disord. 2012;12:12.

\section{Korrespondenz:}

Dr. med. Sima Djalali

Institut für Hausarztmedizin, Universität Zürich

Pestalozzistrasse 24

$\mathrm{CH}-8091$ Zürich

sima.djalali[at]usz.ch

PrimaryResearch - das Fenster zur Forschung

In dieser Artikelserie stellen wir Forschungsarbeiten aus dem Institut für Hausarztmedizin der Universität Zürich (IHAMZ) vor. Die Originalarbeiten sind entweder «open access» zugänglich oder beim jeweiligen Autor auf Anfrage erhältlich. Die Ergebnisse geben einen spannenden Einblick in die täglichen Herausforderungen, aber auch die Leistung der Hausarztmedizin. An dieser Stelle ein herzlicher Dank an alle Kolleginnen und Kollegen, die sich an den Projekten beteiligen und die hier präsentierten Ergebnisse erst ermöglicht haben!

Universität Zürich $^{\text {UzH }}$

Institut für Hausarztmedizin 